\title{
CALCIFICATION OF THE MECONIUM WITHIN THE BOWEL OF THE NEWBORN
}

\author{
BY \\ B. L. HILLCOAT \\ From the Department of Pathology, Brisbane Hospital, Brisbane
}

(RECEIVED FOR PUBLICATION AUGUST 16, 1961)

Since Neuhauser (1944) drew attention to the radiological features of intra-abdominal calcification associated with meconium peritonitis, this condition has been diagnosed with increasing frequency.

However, all cases showing intra-abdominal calcification at birth are not due to meconium peritonitis. Up to the present time four cases have been reported which show calcification within the lumen of the bowel. In all except one, some biochemical investigations were carried out.

Camp and Roberts (1949) reported a baby with ileal stenosis, in whom calcification was present as small concretions in the meconium. No biochemical studies were done on either the baby or the meconium, but radiographs of the skeleton were normal.

Kasmersky and Howard (1952) described a newborn baby with multiple atresias of the jejunum and calcification of the meconium in the bowel between the atretic segments. It was not stated whether meconium was present distal to the last atretic segment or whether each portion of bowel between the atretic segments contained calcified material.

Khilnani, Wolf and Arnheim (1955) reported a case of 'enterolithiasis' in a newborn baby. The pathological features were an imperforate anus, an ectopic right kidney, a deformed right ear, congenital abnormalities of the 11 th and 12 th dorsal vertebrae and calcification of meconium in the lumen of the bowel. Blood calcium estimations on mother and child were normal; the meconium was not analysed.

Rickham (1957) investigated a case of a baby, one month premature, shown radiologically to have calcified intra-abdominal shadows and small gut obstruction. Laparotomy revealed a segment of ileum in which bowel contents were arrested, but no organic obstruction was found. The meconium was washed out and an enterostomy performed. Radiographs of the meconium showed it to be calcified. The condition recurred, and a second operation was necessary, when the proximal intestine was found to be occluded by thick material, and this again proved to be calcified. The baby died, and a hydronephrotic left kidney was found at autopsy. Although trypsin was absent from the first specimen of meconium, trypsin was thereafter present in the duodenal juice and ileal contents. The pancreas was histologically normal, and the baby and mother had normal serum levels of calcium, phosphorus and alkaline phosphatase. Analysis of the meconium showed the phosphorus and calcium to be eight times and five times respectively the normal values given by the author.

In the present report, two additional cases of intraluminal intestinal calcification in the newborn are described.

\section{Case Reports}

Case 1. This baby was born of a 41-year-old mother who had had six normal living children and one miscarriage. Her pregnancy was normal until the 32nd week when an ante-partum haemorrhage occurred. She was admitted to hospital five days later, and labour began after two days. The baby, a male child, was delivered as an assisted breech. He was then noted to have a distended abdomen. Gasping respirations began spontaneously, but death occurred in one and a half hours.

At autopsy no anal orifice or anal dimple was found. It was thought that the limbs were short and the hands of a 'trident' shape. Post-mortem radiographs showed no abnormality of the skeleton, but there was a large number of small circular opacities of varying size throughout the abdomen (Fig. 1). The abdominal cavity revealed gross dilatation of the colon with a blind rectum ending $1 \mathrm{~cm}$. above the anal skin at the level of the prostate gland. On dissection no connexion with the urethra was found. There was no evidence of meconium peritonitis. The small intestine was normal. Other findings were a Meckel's diverticulum $1 \mathrm{~cm}$. long, a large atrial septal defect and small bilateral tears of the tentorium cerebelli with some associated haemorrhage. Radiographs of the bowel containing meconium, of the bowel emptied of meconium, and of the meconium itself (Figs. 2, 3 and 4) showed that the opacities were in the meconium which appeared of normal consistency, though the 'meconium bodies' were a little larger and 


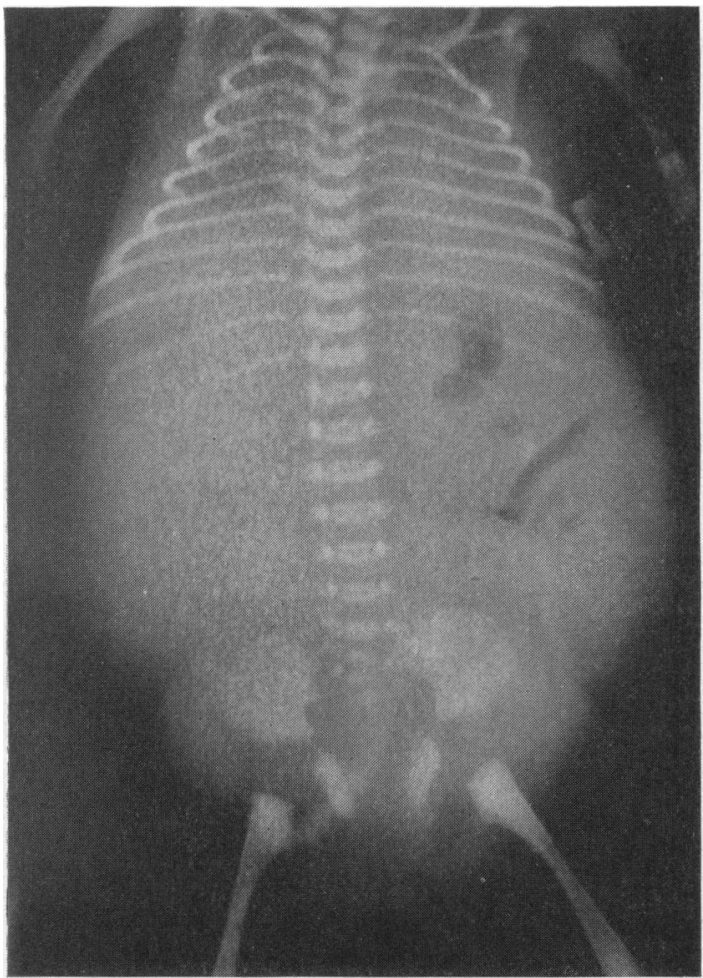

FIG. 1.-Postero-anterior radiograph showing the distended abdomen, a few gas shadows and intraluminal calcification.

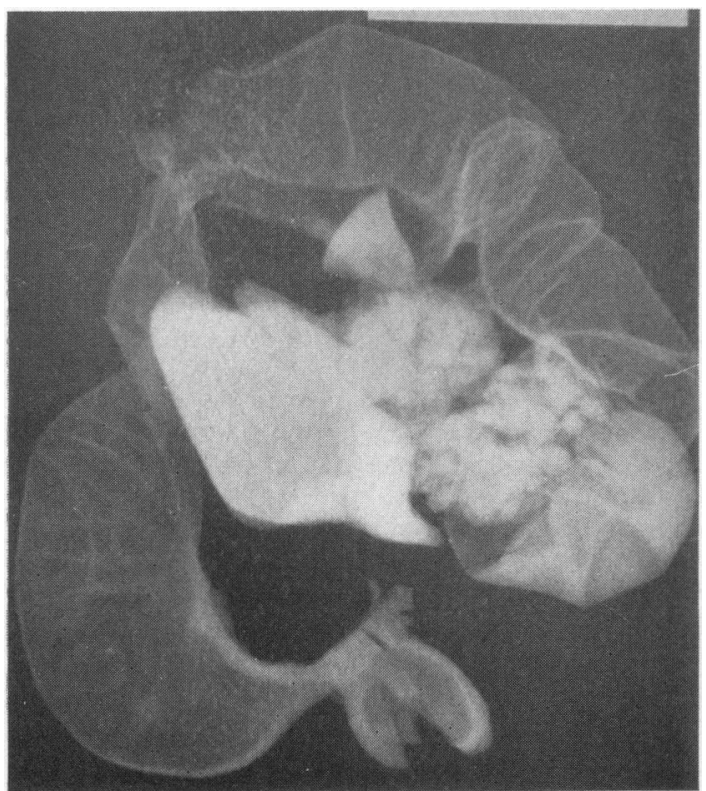

FIG. 3.-Postero-anterior radiograph of intestine emptied of meconium showing absence of calcification and the termination of the blind sac of rectum at the level of the prostate.

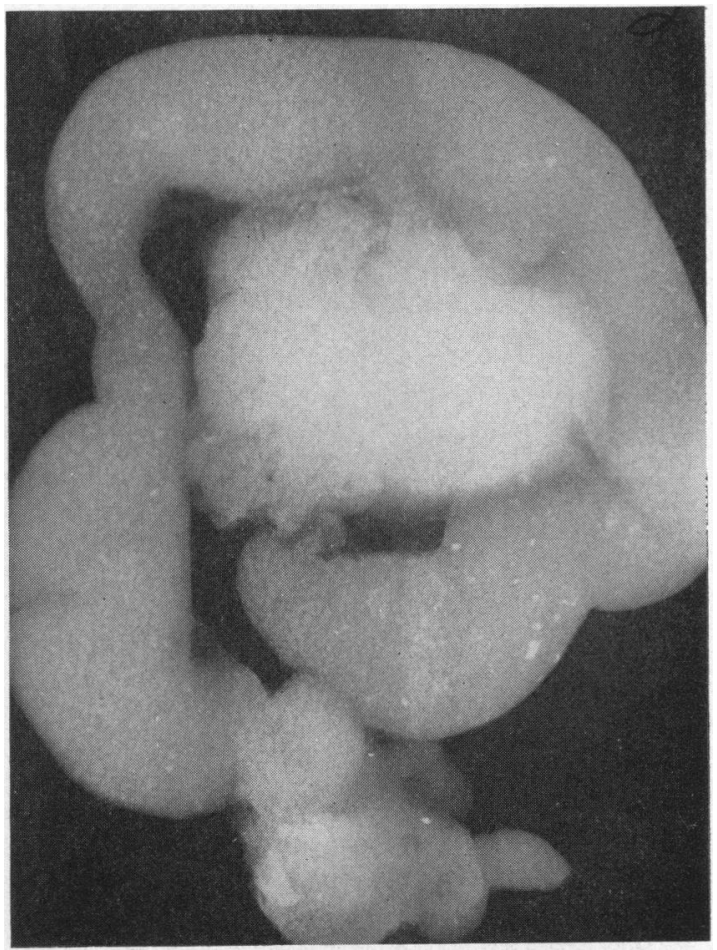

FIG. 2.-Postero-anterior radiograph of intestine containing meconium with intraluminal calcification shown.

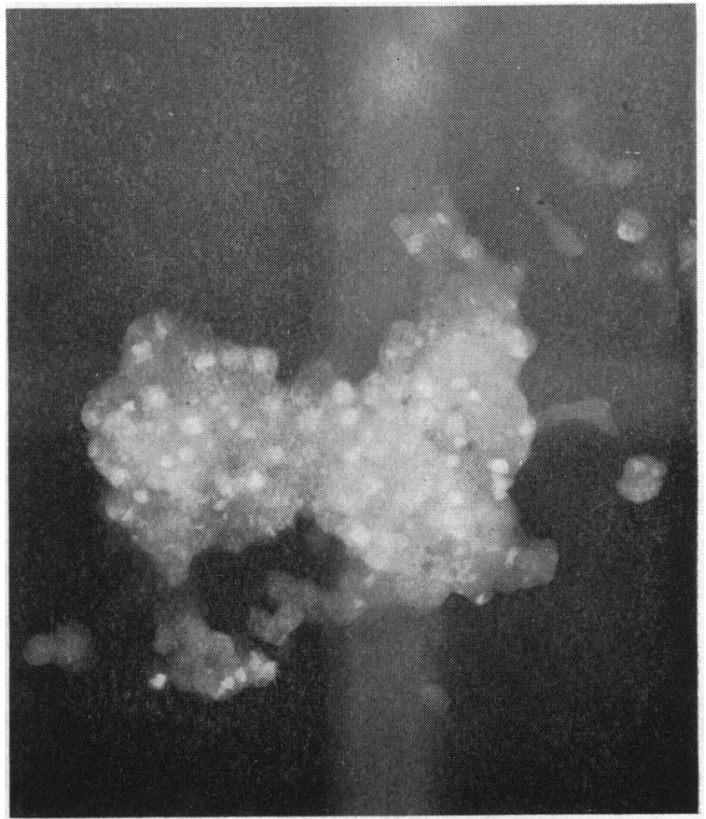

FIG. 4.-Meconium showing calcification. 


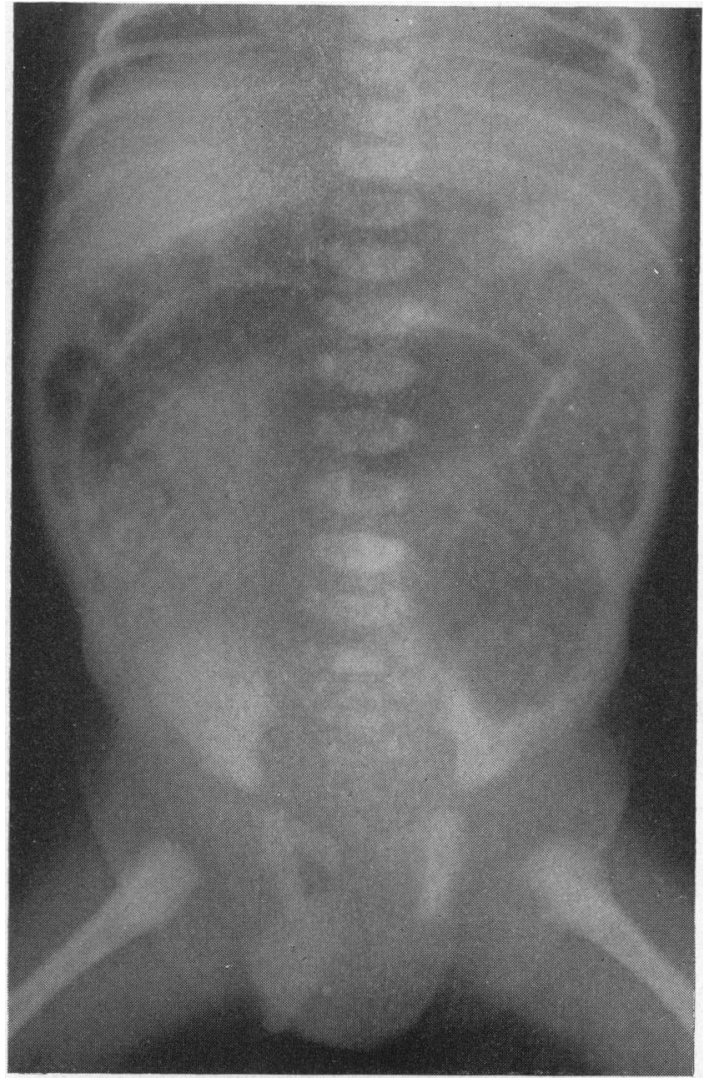

FIG. 5.-Postero-anterior radiograph of abdomen showing distended colon and the circular opacities.

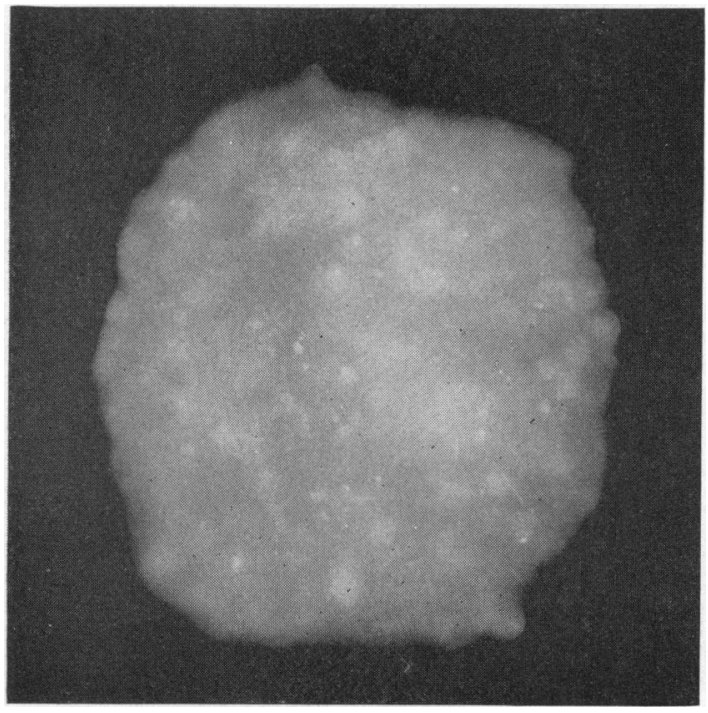

FIG. 6.-Calcification in the meconium concretions. more plentiful than usual. Microscopical examination of the colon showed thinning of the mucosa and submucosa, with the muscle coat normal. The lungs were atelectatic. The parathyroid glands were not examined.

Chemical analysis of the meconium is given in the Table. The baby's mother when questioned had no symptoms of any significance, and estimation of her serum levels of calcium, inorganic phosphate and alkaline phosphatase showed normal levels of calcium $(9.9 \mathrm{mg}$./ $100 \mathrm{ml}$.) and inorganic phosphate $(3.4 \mathrm{mg} . / 100 \mathrm{ml}$. and a low serum alkaline phosphatase value of 2.7 King-Armstrong units (normal range 5-15 KingArmstrong units in this laboratory).

TABLE

ANALYSES OF NORMAL AND ABNORMAL MECONIUM

\begin{tabular}{|c|c|c|c|c|}
\hline & Normal 1 & Normal 2 & Case 1 & Case 2 \\
\hline $\begin{array}{l}\text { Dry Weight }(\%) * \\
\text { Sodium }(\mathrm{g} . \%)+ \\
\text { Potassium }(\mathrm{g} . \%) \dagger \\
\text { Calcium }(\mathrm{g} . \%) \dagger \\
\text { Phosphorus }(\mathrm{g} . \%) \dagger\end{array}$ & $\begin{array}{l}9 \cdot 0 \\
2 \cdot 2 \\
1 \cdot 2 \\
0 \cdot 22 \\
0 \cdot 14\end{array}$ & $\begin{array}{l}18 \\
1 \cdot 8 \\
1 \cdot 44 \\
0 \cdot 2 \\
0 \cdot 08\end{array}$ & $\begin{array}{l}17 \\
0.49 \\
0.13 \\
0.46 \\
0.24\end{array}$ & $\begin{array}{l}17 \\
0 \cdot 1 \\
0 \cdot 44 \\
0 \cdot 68 \\
0 \cdot 24\end{array}$ \\
\hline
\end{tabular}

* Expressed as a percentage of wet weight.

$\dagger$ Expressed as \% dry weight.

Case 2. This fifth baby of a mother of four other normal children was admitted to hospital at the age of $\mathbf{2}$ days with an imperforate anus. Radiographs showed wide separation of the blind end of the rectum and the anal skin, and the presence of intra-abdominal calcification (Fig. 5). Colostomy was done and the meconium obtained proved to be calcified (Fig. 6). There was no evidence of meconium peritonitis. The colostomy functioned well and a repeat $x$-ray examination of the abdomen showed no evidence of recalcification after 26 days. At this stage the child became cyanosed and investigations revealed a congenital heart lesion and the absence of renal function on the right side. The baby improved and was discharged, but required readmission at the age of 3 months because of cardiac failure. This did not respond to treatment and death occurred five days later.

On the first admission the patient's serum calcium was $10.3 \mathrm{mg} . / 100 \mathrm{ml}$., serum inorganic phosphate $5.4 \mathrm{mg} . / 100 \mathrm{ml}$. and serum alkaline phosphatase $9 \cdot 1$ King-Armstrong units. The latter was repeated and a value of $7.9 \mathrm{King}$-Armstrong units obtained. Chemical analysis of the meconium is listed in the Table with that of the previous case, together with chemical analysis of the meconium of two normal babies for comparison.

At autopsy the colostomy was patent and the proximal colon normal. The terminal colon ended in a tapering rectum which was separated by a distance of $3 \mathrm{~cm}$. from the anal skin. There was no recto-vesical or rectourethral fistula. Other findings were agenesis of the right kidney and a total anomalous pulmonary venous drainage. Histologically the small intestine and colon were normal.

The baby's mother, when questioned, had had no 
symptoms of any significance and her serum calcium was $11 \cdot 2 \mathrm{mg}$./100 ml., serum inorganic phosphate $4.2 \mathrm{mg}$./ $100 \mathrm{ml}$. and serum alkaline phosphatase $12 \cdot 1 \mathrm{King}-$ Armstrong units.

\section{Discussion}

The approximate twofold or threefold increase in the calcium and phosphorus in the meconium of the two cases here described is similar to that of the case of Rickham (1957) except that in his case the phosphorus content of the meconium was higher. $\mathrm{He}$ reported also normal levels of serum alkaline phosphatase in mother and child, whereas one baby and one mother in the present cases had low levels of activity of this enzyme. The significance of this observation is unknown.

A striking resemblance exists between the case described by Khilnani et al. (1955) and the two presented here, especially Case 2 , which had, as well as an imperforate anus and calcified meconium, a renal abnormality.

The frequency of intraluminal intestinal calcification in the newborn is difficult to assess. This report brings the total number of cases described to six. Three of these each had an imperforate anus and other abnormalities, one had small bowel atresia, another small bowel stenosis and the sixth a functional small bowel obstruction.

The fact that nearly all babies suspected of having a gut obstruction or an imperforate anus in the neonatal period are examined radiologically, suggests that the phenomenon is uncommon in this group. Its frequency in this group might indeed be equal to its frequency among normal babies. However, this frequency is unknown due to the infrequent abdominal radiography of healthy newborn infants. Further, those babies who are radiographed because of intestinal obstruction or imperforate anus are those who survive the first few hours of extra-uterine life, and this does not include the group who die because of associated abnormalities. Gross (1953) found in a series of 198 babies with imperforate anus that 42 died as a result of an associated abnormality. If these were not radiographed before or after death, intraluminal calcified meconium could easily have been overlooked. Only by chance was it discovered in the first case reported here. It is not known, therefore, whether intestinal obstruction associated with severe abnormalities has a higher frequency of this condition than uncomplicated intestinal obstructions. This could be established by wider use of post-mortem radiography of newborn babies.

Why calcification should occur in intraluminal meconium is unknown. That it occurs readily enough when meconium is free in the peritoneal cavity is well recognized, although this calcification appears to be in irregular plaques in a tissue reaction to the fat in meconium, whereas with intraluminal calcification the calcium appears to be in circular concretions in the meconium. The only evidence of a systemic disturbance is the low serum alkaline phosphatase of one mother and one baby, but neither showed any evidence of hypophosphatasia (Rathbun, 1948). Many factors may influence the serum level of this enzyme so that the significance of low values is difficult to assess.

No theory so far suggested has been proven and none accounts for all the facts observed. Camp and Roberts (1949) point out that calcium and fats are present in meconium, but that the $p \mathrm{H}$ is slightly acid. With disordered physiology of the bowel, a change to alkalinity tends to precipitate calcium, but this, they state, does not explain the many cases of intestinal obstruction without calcification. Kasmersky and Howard (1952) thought that intraluminal calcification occurred in cases of meconium peritonitis and when perforation of the bowel occurred the calcium was deposited throughout the peritoneal cavity. Why the calcification occurs is not shown and the objection stated above still holds. Also intraluminal calcification has not been demonstrated in cases of meconium peritonitis. The theory of Khilnani et al. (1955), who suggest stasis and disordered motility as a possible cause, does not account for the cases of intestinal obstruction without intraluminal calcification. Rickham (1957) admits the aetiology to be unknown and much of the physiology of the foetal gastro-intestinal tract to be unknown also. At the present time this still appears to be true.

\section{Summary}

Two cases of intraluminal intestinal calcification in newborn babies are presented, together with biochemical findings. The four previously reported cases are reviewed and discussed.

No cause has been found to account for the condition.

\section{REFERENCES}

Camp, R. and Roberts, M. H. (1949). Multiple calcareous deposits in the intestinal tract of the newborn: Report of a case associated with stenosis of the ileum. Amer. J. Dis. Child., 78, 393.

Gross, R. E. (1953). The Surgery of Infancy and Childhood; its Principles a:d Techniques. Saunders, Philadelphia.

Kasmersky, C. T. and Howard, W. H. R. (1952). The significance of intra-abdominal calcification in the newborn infant. Amer. J. Roentgenol., 68, 396.

Khilnani, M. T., Wolf, B. S. and Arnheim, E. E. (1955). Enterolithiasis in the newborn. J. Mt Sinai Hosp., 22, 58.

Neuhauser, E. B. D. (1944). The roentgen diagnosis of fetal meconium peritonitis. Amer. J. Roentgenol., 51, 421 .

Rathbun, J. C. (1948). 'Hypophosphatasia': A new developmental anomaly. Amer. J. Dis. Child., 75, 822.

Rickham, P. P. (1957). Intraluminal intestinal calcification in the newborn. Arch. Dis. Childh., 32, 31. 